\title{
Young Egyptians Use of Social Networks and the January 2011 Revolution
}

\author{
Ghada R. El Said \\ Future University in Egypt (FUE) \\ $90^{\text {th }}$ street, Fifth settlement, New Cairo \\ Ghada.refaatafue.edu.eg, \\ Ghadarefaat_04@hotmail.com
}

\begin{abstract}
The 2011 Egyptian protests began on Tuesday 25 January in Tahrir, one of Cairo's biggest squares. On January 25 and 26, the Egyptian government blocked Twitter in Egypt and later Face book was blocked as well.[1] Most observers of the Egyptian scene at that time, claimed that the responsible governmental authorities did this, in an attempt to stop mobilization for anti-government protests.[2]

A report in March 2011[3] highlights a significant increase in the use of the Internet in Egypt in the wake of the January 25 protests. "A large increase in the number of web surfers and users of social networking sites reported to change the pattern of use and the interests of the of the Internet contents". According to the report, the number of Internet users in Egypt prior to January 25 was 21.2 million users, increased by almost $9 \%$ after this date to reach 23.1 million in two months. The time Egyptian users spent online was doubled from 900 to 1800 minutes per months after 25 January 2011. Still, Egypt's Internet penetration rate is less than $25 \%$.

This paper investigates cultural issues in human computer interaction. The paper explores the specific experiences of young Egyptian Internet users and their interaction through social media during and after the Egyptian protest in 25 January 2011. The paper aims to reveal some the cultural characteristics of this user group in interacting with the Internet.
\end{abstract}

Keywords: Internet Social Network, User preference, Culture, language.

\section{Introduction}

In November/ December 2012, the author conducted series of interviews with 45 of young Egyptian Internet users about their experiences with the Internet in general and social media tools in specific. In Egypt, the youth represents $60 \%$ of the overall population, representing the highest majority of Internet users in the country, compared to other age groups. [3]

This paper goes beyond a particular channel to look more broadly at the social network tools use for a group of Egyptians Internet Users, namely during and after the Egyptian protest in 25 January 2011. The paper is examining in what circumstances, 
and why, this group uses social network tools in their computer-mediated communication. In the remainder of this paper, the author will first provide some background information on Internet use in Egypt, then introduce and discuss the study, and finally analyze the results.

\subsection{Technology Context}

It is important here to point a main factor framing this study which is the use of Internet in Egypt. The Internet was first introduced to Egypt in 1993, with a university network. Three years later, the business Internet use began in Egypt with government support through the Information Decision Support Cabinet (IDSC) government entity. Since its start, the Egyptian government placed great emphasis on the Information and Communication technologies (ICT), namely in terms if affordability, availability, and infrastructure and where Egypt is said to have one the fastest growing ICT markets in the world [4].

In 2010, the total number of Internet users in Egypt reached 21.2 million in a population of almost 87 million. This number of users increased by almost $9 \%$ after 25 January 2011 to reach 23.1 million in two months. The time Egyptian users spent online was doubled from 900 to 1800 minutes per months after 25 January 2011. Still, Egypt's Internet penetration rate is less than 25\% [3]. On the other hand, those who are already connected represent the economic middle class and elite, so their influence extends far beyond their somewhat limited numbers, especially in major population centers such as Cairo and Alexandria, the second biggest city in Egypt.

With this context as a background, the exploratory study was conducted with a group of young Internet users in Egypt, namely in Cairo and Alexandria.

\section{Method}

The current research employs semi-structured interviews as an exploratory tool; Interviews are useful for acquiring a wide, yet detailed, picture of a respondent's beliefs about a particular phenomenon. The interviews aimed to find out what the Internet's social network is being used for and what problems, if any, the users experienced, while trying to link the findings with the 2011 Egyptian protests, knowing to be a Face Book revolution. As the Arabic society has been classified as an oral dominant society [5], therefore, the interview was preferred as an appropriate tool for exploratory data collection in this early stage of the research, enabling the researcher to diverge as appropriate from the prepared questions to explore the interesting points that came up. It is planned, on a later stage of the research, to conduct a survey to include more participants.

\subsection{Participants}

The study was carried out among 45 young learners and professionals in Cairo and Alexandria known to be Social Network Internet users. The participants in this study 
are of course not representative of the overall Egyptian population, but they do include among them sample of the protesters who participated in the Egyptian protest in 25 January 2011, known to be "An Egyptian Youth Revolution"[3].

The sample of the study was selected based on convenience sampling through personal contacts of the researcher. The participants were between the ages of 20 and 40 engaged in under-graduate or post-graduate education, and introductory or middle level professional and management positions. All of the participants in the sample uses both Arabic and English languages in online communication. The sample was fairly evenly balanced for gender, with 23young-men and 22 young-women, and teenager. A total of 25 of the people in the sample studied and worked in the information technology field. The remaining 20 studied and worked in a variety of fields such as literature, environmental, and business administration. All of the participants received or currently receiving their education in Egypt, with half of them are studying or studied in English Language.

\section{$2.2 \quad$ Instrument}

Series of semi-structured interview were conducted in different locations in Cairo and Alexandria, during the months of November/ December 2012. The interviews include one question acquiring demographic data (e.g., what is your field of education/ profession?), two general questions about social network tools use (e.g., what is your favorite social network tools, and for how long have you been using it before and after January 2011?), one question about language use online (e.g., what language(s) do you use in online "real-time" posting and chatting with Egyptians and other nationalities?), one question about topic discussed online (e.g., what topic(s) do you discuss online with Egyptians and other nationalities?), two questions about the perceived effect of social network tools in recent social and political changes in Egypt (e.g., do you think that social network tools contributed in any way in recent social and political changes in Egypt, and how?). The interview questions was first pilot tested among three individuals who were not in the in the final interviews, and then finalized and administrated with 45 participants.

The interviews were conducted face-to-face using semi-structured approach, that is, a set of interview questions were planned ahead of time, but the interviews diverged as appropriate to explore interesting points that came up. Each interview session lasted from 60 to 75 minutes and was tape recorded with the consent of the participants.

\subsection{Data Analysis}

The interviews were transcribed and analyzed through textual analysis and the written transcripts were examined by an individual judge, to identify patterns. Textual analysis involves coding participants' answers, and classifying words under main groups. The frequency of the answers helped to identify patterns and relationships and to distinguish differences and similarities within responses. 


\section{$3 \quad$ Results}

The analysis of the interview data reveals some interesting findings about the way in which Egyptian users interact with the Internet's social network in general, and before and after January 2011 specifically.

The first general finding is that English is the language with which all interview's participants reported they interact online. This suggests a change in the language used on line for the Egyptians, contradicting with previous research, monitoring the Egyptians use of Internet over the last decade. In 1999, an early investigation of the adaption of Internet in Egypt suggested that language is one of the main barriers to the adoption of Internet in Egypt [6]. In 2001, another exploratory study recommended that Egyptians, in social communication online, use either Arabic or what is known as 'Romanized Arabic', here Latin characters are used to approximate Arabic words, in social Internet communication [7].

The second result is related to the change of Internet usage context and tools, for the target sample, before and after January 2011. The early investigational studies on online activities for Internet users in Egypt, conducted in the years 2000 and 2001 $[6,7]$ reported that social communication and entertainment are the most popular use context of the Internet in Egypt. It seems that this was the case until early 2011!

The highest majority (90\%) of the interviews' participants in the current study reported that before January 2011, they mainly used Face book for social communications with friends. After the Egyptian revolution in January 2011, and with the political fast changes happening in the country to date, they became Political News Seekers on Twitter and U-Tube. While the highest majority of the interviews' participants reported that they on daily basis seek political opinions and explanations from Egyptians political faces twits; they also cited that they regularly watch political talkshows on U-Tube. ".....I use to use Face book for chat with friends before the revolution. Now me and most of my friends, we are daily users of Twitter and U-Tube, as it is faster, we seek political opinions and explanation from political leaders we trust, either by following politicians on Twitter, or by watching political talk-shows on U-Tube...." as expressed one of the interviewees. "The revolutions is still ongoing, things move fast in Egypt, many events are not comprehensive to youth like us.... we continuously seek explanation of what is happening from political faces who are now all on Twitter", as cited by Amr Mansour, a 21 years-old Egyptian graduate who was never interested in political issues prior 2011, but now is involved of political news and updates on the Internet.

The third result is related to the preference of text-based interpersonal communication. Previous social and cultural studies on preferences of Arab communication patterns, conducted in 1995 [5] reported that, due to their oral dominant culture, Arabic computer users may find text-based interfaces lacking when it comes to interpersonal communication. It seems that this might not be the case anymore.

The highest majority $(87 \%)$ of the interviews' participants in the current study reported their preference for Internet text-based communication through social networks. "It is faster, affordable, and more practical", as cite by most of the interviewees. "During the old regime, Egyptians were unable to know the reality and 
express themselves freely because the media was controlled. Social networks helped to exchange opinions on what was happening and to mobilize people through face book pages such as Khaled Said and 6 April Movement... people exchange written comments, articles and published news", as expressed by one interviewee.

\section{Discussion}

The preliminary analysis of interviews suggests a difference in the online behavior of Egyptians users in 2011 as they were before that date.

Firstly concerning use of language online: While all participants at the current exploratory study reported use of English language in all of their Internet communication, this is contradicting results of previous studies investigated language choice on line for Egyptian users in 1999 and 2001[6,7], and suggested that Arabic is dominant in communication on line for this cultural group. This suggested change in online behavior was anticipated by a study which was conducted in Egypt in 2007 and looked at language choice online in light of globalization and identity issues [9]. The study predicted that in Egypt, as in many other parts of the world, Internet will be having a great impact on language use; with a global use of English on line to such degree that other languages would be crowded out.

It worth mention that although English has been always the principal foreign language of the general population in Egypt and it is the only mandatory foreign language taught in schools in Egypt. It is only in 2004, English started to be mandatory taught in primary public schools, with obligatory instruction starting in first grade.

Secondly concerning the context of use of the Internet: According to the highest majority of interviewees, prior the Egyptian revolution, they were more interested in entertainment, but after the revolution they are now more interested in searching for credible news and finding sources for live political updates and follow-up. Though only a fraction of Egyptians have Internet access, "Through blogs, Twitter and Face book, the Web has become a haven for a young, educated class yearning to express its worries and anxieties" as expressed by a number of interviewees.

Thirdly concerning text-based interpersonal communication: According to the highest majority of interviewees, text-based interpersonal communication through the Internet is now preferable over oral communication through mobiles and phones. This finding is contradicting with what is known about Arabic society which has been classified as an oral dominant society. Previous studies looking at cultural preferences of Arab communication patterns ${ }^{[5]}$ suggested that oral messages are more valued than written messages for their affective power in interpersonal association between communicators.

\section{Conclusion}

The social network tools are bound to have an important long-term effect on users' communication, empowering, and language use -which is the most salient cultural variable. The trends discussed in this paper can suggest that social change can be 
caused by Internet media. As some research [9] pointed out, the major social dynamic shaping international media and communication is the contradiction between global networks and local identities. In that light, it is worthwhile to investigate in more depth whether the online use of English and the shift from oral to text communication by this small group of young Egyptians might reflect broader and long-term social and cultural shifts.

\section{$6 \quad$ Limitation}

It is important to point out the limitation of this study. The sample size is relatively small and was selected through convenient sampling through personal contact of the researchers, and is thus non-random. The fact that the participants were known by the researcher may have affected people's answers. The interview items were explored for patterns and illustrative examples rather than systematically coded. No validity or reliability tests were done for the interviews items. Therefore, the results of the study cannot be generalizable to other populations beyond this group of participants. On the other hand, this study would be considered an exploratory investigation that had the goal of identifying possible issue and trends for further research.

\section{References}

1. Twitter blocked in EgyptAmidst Rising Protests. TechCrunch (January 25, 2011), http: / / techcrunch.com/2011/01/25/twitter-blocked-egypt /

2. Egypt severs internet connection amid growing unrest. BBC News (January 28, 2011), http: / /www.bbc.co.uk/news/technology-12306041

3. A significant increase in the number of Internet users in Egypt after the January Uprising. Reuters (March 19, 2011), http: / / mymobimobi .com/en-lang/viewNews / 62

4. IT in Egypt, Cairo American Chamber of Commerce in Egypt (1998)

5. Zaharna, R.: Understanding Cultural Preferences of Arab Communication Patterns. Public Relations Review 21(3), 241-255 (1995)

6. El Nawawy, M.: Profiling Internet Users in Egypt: Understanding the Primary Deterrent against Their Growth in Number. In: The Tenth Internet Society (ISOC) Annual Conference: INET 2000 CD Proceedings, Yokohama, Japan, July 18-21 (2000)

7. El Said, G.R., Hone, K.S.: Use of Card Sorting for Cultural Web-Preferences Data Elicitation: The Case of Egyptian Internet Users. In: Stephanidis, C. (ed.) Universal Access in HCI: Inclusive Design in the Information Society, June 22-27. The Tenth International Conference on Human-Computer Interaction, HCI International' 2003 Proceedings, vol. 4. Lawrence Erlbaum Associates Publishers (2003) ISBN: 0-8058-4933-5

8. Warschauer, M., El Said, G.R., Zohry, A.: Language Choice Online: Globalization and Identity in Egypt. In: Danet, B., Herring, S. (eds.) The Multilingual Internet: Language, Culture and Communication Online, pp. 303-318. Oxford University Press (2007) ISBN: 978-0-19-530479-4

9. Friedman, T.: The sociolinguistic market of Cairo: Gender, class, and education. Kegan Paul International, London (1999) 